PROCEEDINGS OF THE

AMERICAN MATHEMATICAL SOCIETY

Volume 127, Number 11, Pages 3161-3167

S 0002-9939(99)04926-6

Article electronically published on May 4, 1999

\title{
NOETHERIAN DOWN-UP ALGEBRAS
}

\author{
ELLEN KIRKMAN, IAN M. MUSSON, AND D. S. PASSMAN \\ (Communicated by Lance W. Small)
}

\begin{abstract}
Down-up algebras $A=A(\alpha, \beta, \gamma)$ were introduced by G. Benkart and $\mathrm{T}$. Roby to better understand the structure of certain posets. In this paper, we prove that $\beta \neq 0$ is equivalent to $A$ being right (or left) Noetherian, and also to $A$ being a domain. Furthermore, when this occurs, we show that $A$ is Auslander-regular and has global dimension 3.
\end{abstract}

\section{$\S 1$. INTRODUCTION}

Motivated by the study of posets, G. Benkart and T. Roby introduced certain down-up algebras in [BR], see also [B]. Specifically, let $K$ be a field, fix parameters $\alpha, \beta, \gamma \in K$ and let $A=A(\alpha, \beta, \gamma)$ be the $K$-algebra with generators $d$ and $u$, and relations

$$
\begin{aligned}
& d^{2} u=\alpha d u d+\beta u d^{2}+\gamma d=(\alpha d u+\beta u d+\gamma) d \\
& d u^{2}=\alpha u d u+\beta u^{2} d+\gamma u=u(\alpha d u+\beta u d+\gamma)
\end{aligned}
$$

Note that

$$
(u d)(d u)=u\left(d^{2} u\right)=u(\alpha d u+\beta u d+\gamma) d=\left(d u^{2}\right) d=(d u)(u d),
$$

by (1.1) and (1.2), and therefore $u d$ and $d u$ commute in $A$. Furthermore, $A$ is clearly isomorphic to its opposite ring $A^{\mathrm{op}}$ via the map $d \mapsto u^{\mathrm{op}}$ and $u \mapsto d^{\mathrm{op}}$.

Our main result here is

Theorem. If $A=A(\alpha, \beta, \gamma)$, then the following are equivalent.

(1) $\beta \neq 0$.

(2) A is right (or left) Noetherian.

(3) A is a domain.

(4) $K[u d, d u]$ is a polynomial ring in the two generators.

Furthermore, if these conditions hold, then $A$ is Auslander-regular and has global dimension 3 .

In particular, we answer some questions posed in a preliminary version of [B]. Note that condition (4) above is significant because $K[u d, d u]$ plays the role of the enveloping algebra of a Cartan subalgebra in the highest weight theory of [BR].

Received by the editors January 28, 1998.

1991 Mathematics Subject Classification. Primary 16E70, 16P40.

This research was supported in part by NSF Grants DMS-9500486 and DMS-9622566.

(C)1999 American Mathematical Society 
As is apparent, all our positive results for $A$ occur when the parameter $\beta$ is not zero. In this situation, we offer two distinct approaches to the study of $A$, namely via filtered rings and via generalized Weyl algebras.

\section{§2. The generalized Weyl algebra approach}

2.1. Suppose that $\beta \neq 0$. We first show that $A$ embeds in a skew group ring. To this end, let $R=K[x, y]$ be a polynomial ring in two variables, and define $\sigma \in \operatorname{Aut}_{K}(R)$ by $\sigma(x)=y$ and $\sigma(y)=\alpha y+\beta x+\gamma$. Note that $\sigma$ is indeed an automorphism since $\beta \neq 0$, and we can form $S=R\left[z, z^{-1} ; \sigma\right]$, the skew group ring of the infinite cyclic group $\langle z\rangle$ over $R$, with $r z=z \sigma(r)$ for all $r \in R$. Now consider the elements $D=z^{-1}$ and $U=x z$ in $S$. Then $U D=x z z^{-1}=x$ and $D U=z^{-1} x z=\sigma(x)=y$. In addition,

$$
\begin{aligned}
D^{2} U & =D \cdot D U=z^{-1} y=\sigma(y) z^{-1} \\
& =(\alpha y+\beta x+\gamma) z^{-1}=(\alpha D U+\beta U D+\gamma) D
\end{aligned}
$$

and

$$
\begin{aligned}
D U^{2} & =D U \cdot U=y x z=x z \sigma(y) \\
& =U(\alpha y+\beta x+\gamma)=U(\alpha D U+\beta U D+\gamma) .
\end{aligned}
$$

Hence, there is an algebra homomorphism $\theta: A(\alpha, \beta, \gamma) \rightarrow S$ given by $\theta(d)=D$ and $\theta(u)=U$. Since $\theta(u d)=U D=x$ and $\theta(d u)=D U=y$ are algebraically independent, the same is true of the commuting elements $u d$ and $d u$. In particular, $K[u d, d u]$ is isomorphic to a polynomial ring in the two variables and $\sigma$ lifts to an automorphism of this algebra satisfying $\sigma(u d)=d u$ and $\sigma(d u)=\alpha d u+\beta u d+\gamma$.

It remains to show that $\theta$ is a monomorphism. To this end, note that (1.1) implies

$$
\begin{aligned}
& d(u d)=(d u) d=\sigma(u d) d, \\
& d(d u)=(\alpha d u+\beta u d+\gamma) d=\sigma(d u) d,
\end{aligned}
$$

and similarly, (1.2) yields

$$
\begin{aligned}
& (d u) u=u(\alpha d u+\beta u d+\gamma)=u \sigma(d u), \\
& (u d) u=u(d u)=u \sigma(u d) .
\end{aligned}
$$

Thus

$$
d c=\sigma(c) d, \quad c u=u \sigma(c) \quad \text { for all } c \in K[u d, d u],
$$

and therefore $d K[u d, d u]=K[u d, d u] d$ and $K[u d, d u] u=u K[u d, d u]$. In particular, if we set

$$
B=\sum_{k \geq 0} K[u d, d u] d^{k}+\sum_{k \geq 0} K[u d, d u] u^{k+1} \subseteq A,
$$

then $d B \subseteq B$ and $u B \subseteq B$. Hence $A B \subseteq B$ and consequently $A=B$. In other words, $A$ is spanned by the set $\mathcal{B}=\left\{(u d)^{i}(d u)^{j} d^{k},(u d)^{i}(d u)^{j} u^{k+1} \mid i, j, k \geq 0\right\}$. But $\theta(\mathcal{B})=\left\{x^{i} y^{j} z^{-k}, x^{i} y^{j}(x z)^{k+1} \mid i, j, k \geq 0\right\}$, and these elements are clearly linearly independent in $S$. It follows that $\mathcal{B}$ is a basis for $A$ and that $\theta$ is indeed a one-to-one map.

Corollary. If $\beta \neq 0$, then $K[u d, d u]$ is a polynomial ring in the two generators and $A(\alpha, \beta, \gamma)$ is a domain. 
2.2. If $R$ is any $K$-algebra, $\sigma$ any $K$-automorphism of $R$ and $x$ any central element of $R$, then the generalized Weyl algebra $R(\sigma, x)$ is defined to be the algebra generated by $R$ and the two variables $X^{+}$and $X^{-}$subject to the relations

$$
\begin{gathered}
X^{-} X^{+}=x, \quad X^{+} X^{-}=\sigma(x), \\
X^{+} r=\sigma(r) X^{+}, \quad X^{-} \sigma(r)=r X^{-} \quad \text { for all } r \in R .
\end{gathered}
$$

Here, we take $R=K[x, y]$ and we let $\sigma$ be described as in the preceding section. Then $\sigma(x)=y, \sigma(y)=\alpha y+\beta x+\gamma$, and it follows from the above that

$$
\begin{aligned}
X^{+}\left(X^{+} X^{-}\right) & =X^{+} y=\sigma(y) X^{+}=(\alpha y+\beta x+\gamma) X^{+} \\
& =\left(\alpha X^{+} X^{-}+\beta X^{-} X^{+}+\gamma\right) X^{+}
\end{aligned}
$$

and

$$
\begin{aligned}
\left(X^{+} X^{-}\right) X^{-} & =y X^{-}=X^{-} \sigma(y)=X^{-}(\alpha y+\beta x+\gamma) \\
& =X^{-}\left(\alpha X^{+} X^{-}+\beta X^{-} X^{+}+\gamma\right) .
\end{aligned}
$$

Thus there exists an algebra homomorphism $\varphi: A \rightarrow R(\sigma, x)$ given by $d \mapsto X^{+}$and $u \mapsto X^{-}$. On the other hand, (2.5) implies that the map $\varphi^{\prime}: R(\sigma, x) \rightarrow A$ given by $X^{+} \mapsto d$ and $X^{-} \mapsto u$ is also an algebra homomorphism. Therefore $\varphi^{\prime}=\varphi^{-1}$ and $\varphi$ is an isomorphism. In other words, we have shown

Theorem. If $\beta \neq 0$, then the algebra $A=A(\alpha, \beta, \gamma)$ is isomorphic to a generalized Weyl algebra $R(\sigma, x)$ with $R=K[x, y]$.

Consequently, [Bv1, Proposition 7] yields

Corollary. If $\beta \neq 0$, then $A(\alpha, \beta, \gamma)$ is right and left Noetherian.

\section{$\S 3$. ThE FILTERED RING APPROACH}

3.1. To start with, let $\alpha, \beta, \gamma$ be arbitrary parameters and let $A=A(\alpha, \beta, c)$. We define a filtration on $A$ for which the associated graded ring is isomorphic to $A(\alpha, \beta, 0)$. To this end, let $V=K+K u+K d$ and let $V_{n}=V^{n}$. Then $V_{0}=K$, $V_{1}=V$, and $\left\{V_{n} \mid n=0,1,2, \ldots\right\}$ is obviously a filtration of $A$. Certainly, $\bar{u}=u+K$ and $\bar{d}=d+K$ generate the associated graded ring $\mathrm{Gr} A$, and it is clear, from (1.1) and (1.2), that $\bar{u}$ and $\bar{d}$ satisfy the generating relations of $A(\alpha, \beta, 0)$. Thus, there exists an epimorphism $\rho: A(\alpha, \beta, 0) \rightarrow$ Gr $A$ given by $U \mapsto \bar{u}$ and $D \mapsto \bar{d}$. Here, of course, we use $U$ and $D$ to denote the obvious generators of $A(\alpha, \beta, 0)$. To see that $\rho$ is an isomorphism, we use the PBW Theorem for downup algebras as given in [B, Theorem 4.1]. Specifically, that result asserts that $\mathcal{C}=\left\{u^{i}(d u)^{j} d^{k} \mid i, j, k \geq 0\right\}$ is a basis for $A$ and that $V_{n}=V^{n}$ is spanned by those monomials with $i+2 j+k \leq n$. With this observation, it is clear that $V_{n} / V_{n-1}$ has basis $\mathcal{C}_{n}=\left\{\bar{u}^{i}(\bar{d} \bar{u})^{j} \bar{d}^{k} \mid i, j, k \geq 0, i+2 j+k=n\right\}$, and hence $\overline{\mathcal{C}}=\bigcup_{n=0}^{\infty} \mathcal{C}_{n}$ is a basis of $\operatorname{Gr} A$. But $\left\{U^{i}(D U)^{j} D^{k} \mid i, j, k \geq 0\right\}$ is a basis of $A(\alpha, \beta, 0)$, by [B, Theorem 4.1] again, and $\rho$ maps this basis to $\overline{\mathcal{C}}$. Consequently, $\rho$ is one-to-one, and we have shown

Lemma. $A=A(\alpha, \beta, \gamma)$ has a filtration whose associated graded ring is isomorphic to $A(\alpha, \beta, 0)$. 
3.2. We now show that if $\beta \neq 0$ and if $K$ is sufficiently big, then the algebra $A(\alpha, \beta, 0)$ is an iterated Ore extension. To start with, fix nonzero elements $\lambda, \mu \in K$, and let $B$ be the algebra with generators $a$ and $b$, and with relation

$$
b a=\mu a b .
$$

Then $B$ is clearly an Ore extension of its polynomial subalgebra $K[a]$, and hence $\left\{a^{i} b^{j} \mid i, j \geq 0\right\}$ is a basis of $B$. Now let $\tau$ be the automorphism of $B$ defined by $\tau(a)=\lambda a, \tau(b)=\mu b$, and let $\delta: B \rightarrow B$ be the $K$-linear map determined by

$$
\delta\left(a^{m} b^{n}\right)=p_{m}(\lambda, \mu) a^{m-1} b^{n+1} \quad \text { for all } m, n \geq 0,
$$

where

$$
p_{m}=p_{m}(\lambda, \mu)=\sum_{i=0}^{m-1} \lambda^{i} \mu^{m-1-i} .
$$

Since $p_{m+t}=\mu^{t} p_{m}+\lambda^{m} p_{t}$, it is easy to see that $\delta$ is a $\tau$-derivation of $B$, that is,

$$
\delta(r s)=\delta(r) s+\tau(r) \delta(s) \quad \text { for all } r, s \in B .
$$

Hence, we can form the Ore extension $C=B[c ; \tau, \delta]=C(\lambda, \mu)$. Basic properties of such extensions can be found in [GW, Chapter 1]. In particular, $C$ is a free left and right $B$-module with basis $\left\{c^{i} \mid i \geq 0\right\}$, and with multiplication determined by

$$
c r=\tau(r) c+\delta(r) \quad \text { for all } r \in B .
$$

Indeed, since $\delta(a)=b$ and $\delta(b)=0$, we have

$$
c a=\lambda a c+b, \quad c b=\mu b c .
$$

Furthermore, $C$ has a basis over $K$ consisting of all monomials $a^{i} b^{j} c^{k}$ with $i, j, k \geq 0$, and it is clear that $C=C(\lambda, \mu)$ is the $K$-algebra generated by $a, b$ and $c$ subject to the relations (3.1) and (3.2).

3.3. If $\eta \in K$ and if $r$ and $s$ are elements of any $K$-algebra, we introduce the notation $[r, s]_{\eta}=r s-\eta s r$. Now suppose that $\beta \neq 0$. If $\alpha^{2}+4 \beta$ is a square in $K$, we say that $A=A(\alpha, \beta, \gamma)$ is split, and we can let $\lambda$ and $\mu$ be the roots of the quadratic equation

$$
\zeta^{2}-\alpha \zeta-\beta=0
$$

Thus $\lambda+\mu=\alpha, \lambda \mu=-\beta$, and $\lambda, \mu \neq 0$ since $\beta \neq 0$.

Now it is easily seen that the defining relations (1.1) and (1.2) for $A(\alpha, \beta, 0)$ can be expressed in the form

$$
\left[[D, U]_{\lambda}, U\right]_{\mu}=0=\left[D,[D, U]_{\lambda}\right]_{\mu} .
$$

Indeed,

$$
\begin{aligned}
{\left[D,[D, U]_{\lambda}\right]_{\mu} } & =[D, D U-\lambda U D]_{\mu}=D(D U-\lambda U D)-\mu(D U-\lambda U D) D \\
& =D^{2} U-(\lambda+\mu) D U D+\lambda \mu U D^{2}=D^{2} U-\alpha D U D-\beta U D^{2},
\end{aligned}
$$

so $0=\left[D,[D, U]_{\lambda}\right]_{\mu}$ is equivalent to (1.1), and similarly, $\left[[D, U]_{\lambda}, U\right]_{\mu}=0$ is equivalent to relation (1.2).

Finally, if we set $H=[D, U]_{\lambda}$, then (3.3) translates to

$$
H U=\mu U H, \quad D H=\mu H D, \quad D U=\lambda U D+H .
$$

In other words, $A(\alpha, \beta, 0)$ is generated by the elements $U, D$ and $H$ subject to the relations (3.4) and, in view of the comment at the end of $\S 3.2$, there is an algebra 
isomorphism $\psi: C \rightarrow A(\alpha, \beta, 0)$ given by $\psi(c)=D, \psi(a)=U$ and $\psi(b)=H$. By combining all of this, we have therefore proved

Theorem. Assume that $A=A(\alpha, \beta, \gamma)$ is split and that $\beta \neq 0$. Then $A$ has a filtration whose associated graded ring $\mathrm{Gr} A \cong A(\alpha, \beta, 0)$ is isomorphic to an iterated Ore extension of the form $K[a][b ; \eta][c ; \tau, \delta]$.

We can also use the above result to prove that $A=A(\alpha, \beta, \gamma)$ is Noetherian when $\beta \neq 0$. Indeed, for this it suffices to extend the field and assume that $A$ is split. Then $\operatorname{Gr} A$ is an iterated Ore extension, so Gr $A$ is right and left Noetherian, and hence so is $A$.

\section{$\S 4$. MAin RESUlts}

4.1. We start by considering the global dimension of $A=A(\alpha, \beta, \gamma)$. Since $A$ and its associated graded ring $\mathrm{Gr} A \cong A(\alpha, \beta, 0)$ are both isomorphic to their opposite rings, left and right global dimensions are equal here. Thus we can use gl dim to denote this common dimension.

Theorem. If $\beta \neq 0$, then $\operatorname{gl} \operatorname{dim} A(\alpha, \beta, \gamma)=3$.

Proof. We first show that $\operatorname{gl} \operatorname{dim} A<\infty$ and for this, it suffices to assume that $A$ is split. Indeed, if $F$ is a field extension of $K$, then $A^{F}=F \otimes A$ is a free $A$-module and hence $\mathrm{gl} \operatorname{dim} A \leq \mathrm{gl} \operatorname{dim} A^{F}$ by [McR, Theorem 7.2.8]. Now if $A$ is split, then Theorem 3.2 implies that $A$ has a filtration with $\operatorname{Gr} A$ isomorphic to an iterated Ore extension. Thus gl $\operatorname{dim} \operatorname{Gr} A<\infty$ by [McR, Theorem 7.5.3], and consequently gl $\operatorname{dim} A<\infty$ by [McR, Corollary 7.6.18].

Now, by Theorem 2.2, $A$ is isomorphic to a generalized Weyl algebra $R(\sigma, x)$ with $R=K[x, y]$. Here the automorphism $\sigma$ of $R$ is given by $\sigma(x)=y$ and $\sigma(y)=\alpha y+\beta x+\gamma$. Thus the maximal ideals $Q=(x, y)$ and $P=\sigma^{-1}(Q)$ of $R$ both contain $x$, and it follows from [Bv2, Theorem 3.7] that $\mathrm{gl} \operatorname{dim} A=3$.

4.2. Recall that a Noetherian ring $R$ is said to be Auslander-regular if $R$ has finite global dimension and if, for every finitely generated $R$-module $M$ and positive integer $q$, we have $j(N) \geq q$ for every submodule $N$ of $\operatorname{Ext}_{R}^{q}(M, R)$. Here $j(M)=$ $\min \left\{j \mid \operatorname{Ext}_{R}^{j}(M, R) \neq 0\right\}$. Furthermore, $R$ is said to be Cohen-Macaulay if $R$ has finite GK-dimension, and if the equality $\operatorname{GKdim} M+j(M)=\operatorname{GKdim} R$ holds for every finitely generated $R$-module $M$.

Lemma. Let $\beta \neq 0$ and write $A=A(\alpha, \beta, \gamma)$.

(i) A is Auslander-regular.

(ii) If $A$ is split, then it is also Cohen-Macaulay.

Proof. (i) As is shown in [Bv2, pp. 88-89], a generalized Weyl algebra $R(\sigma, x)$ is always a factor ring of an iterated skew polynomial extension of $R$. The argument is as follows. First form the polynomial ring $R[z]$ and consider the generalized Weyl algebra $R[z](\sigma, x+z)$, where $\sigma$ is extended to $R[z]$ by taking $\sigma(z)=z$. Thus, since $z$ is in the center of $R[z](\sigma, x+z)$ and since $R[z](\sigma, x+z) /(z) \cong R(\sigma, x)$, it suffices to show that $R[z](\sigma, x+z)$ is an iterated skew polynomial ring extension of $R$. For this, note that $R[z](\sigma, x+z) \cong R\left[X^{-} ; \sigma^{-1}\right]\left[X^{+} ; \sigma, \delta\right]$, where the automorphism $\sigma$ is extended to $R\left[X^{-} ; \sigma^{-1}\right]$ by $\sigma\left(X^{-}\right)=X^{-}$, and the $\sigma$-derivation $\delta$ is defined by $\delta(r)=0$ for all $r \in R$ and $\delta\left(X^{-}\right)=\sigma(x)-x$. 
We now proceed to show that $A$ is Auslander-regular. To start with, Lemma 3.1 and $[\mathrm{Bj}$, Theorem 4.1] allow us to assume that $\gamma=0$. Consequently, $\sigma$ is a graded automorphism of $R=K[x, y]$ and hence the Ore extensions above are all constructed via graded automorphisms. In other words, $R[z](\sigma, x+z)$ is an iterated Ore extension of a connected graded $K$-algebra, with each automorphism graded. Thus, [GZ, Lemma 3.8(2)] implies that $R[z](\sigma, x+z)$ is Auslander-regular (and also Cohen-Macaulay). But $z$ is a central regular element, so we can conclude from $[\mathrm{L}, \S 3.4$ Remark (3)] that the Auslander condition carries over to the factor ring $R[z](\sigma, x+z) /(z) \cong R(\sigma, x) \cong A$.

(ii) Now suppose that $A$ is split. Then, by Lemma 3.1, $A$ has a filtration with $A_{0}=K$ and with $\operatorname{Gr} A \cong A(\alpha, \beta, 0)$. Hence, by [GZ, Lemma 3.8(1)], it suffices to show that $A(\alpha, \beta, 0)$ is Auslander-regular and Cohen-Macaulay. To this end, observe that $A(\alpha, \beta, 0) \cong K[a][b ; \eta][c ; \tau, \delta]$, by Theorem 3.3 , where $\eta$ and $\tau$ are graded algebra automorphisms. Furthermore, in each Ore extension, the set of elements of total degree 0 is precisely equal to $K$. Thus, [GZ, Lemma 3.8(2)] implies that $A(\alpha, \beta, 0)$ is Auslander-regular and Cohen-Macaulay, as required.

4.3. Lemma. If $\beta=0$, then $A$ is not right or left Noetherian.

Proof. For convenience, set $x=d u$ so that $\left\{u^{i} x^{j} d^{k} \mid i, j, k \geq 0\right\}$ is a $K$-basis for $A$. Since $\beta=0$, (1.1) yields $d x=(\alpha x+\gamma) d$ and hence $d x^{j}=(\alpha x+\gamma)^{j} d$ for all $j \geq 0$. Furthermore, by (1.2), we have $(\alpha u d+\gamma-x) u=0$.

For each $n \geq 0$, set

$$
I_{n}=\sum_{i=0}^{n} u^{i}(\alpha u d+\gamma-x) A .
$$

Then, since $(\alpha u d+\gamma-x) u=0$, we have

$$
I_{n}=\sum_{i=0}^{n} \sum_{j, k=0}^{\infty} K u^{i}(\alpha u d+\gamma-x) x^{j} d^{k} .
$$

In particular, since $u d x^{j} d^{k}=u(\alpha x+\gamma)^{j} d^{k+1}$, it follows that no element of $I_{n}$ can contain the monomial $u^{n+1} x$ in its support. Thus $u^{n+1}(\alpha u d+\gamma-x) \notin I_{n}$ and hence $I_{n+1}$ is properly larger than $I_{n}$. In other words, we have shown that $I_{0} \subseteq I_{1} \subseteq I_{2} \subseteq \cdots$ is a properly increasing sequence of right ideals of $A$, and therefore $A$ is not right Noetherian. Since $A \cong A^{\text {op }}$, the result follows.

4.4. Proof of the main theorem. Suppose first that $\beta \neq 0$. Then, by Corollary $2.1, A$ is a domain and $K[u d, d u]$ is a polynomial ring in the two generators. Furthermore, Corollary 2.2 implies that $A$ is right and left Noetherian, Theorem 4.1 yields the appropriate information on the global dimension of $A$, and Lemma 4.2 asserts that $A$ is Auslander-regular.

Conversely, suppose $\beta=0$. Then $A$ is not a domain since, as observed in [B], $d(d u-\alpha u d-\gamma)=0$. Futhermore, multiplying this relation on the left by $u$ shows that $d u$ and $u d$ are algebraically dependent. Finally, $A$ is not left or right Noetherian by Lemma 4.3 .

We remark in closing that the recent manuscript $[\mathrm{Z}]$ completely determines the center of $A(\alpha, \beta, \gamma)$, while $[\mathrm{K}]$ proves the equivalence of (1) and (3) by showing that $A$ is a hyperbolic ring if $\beta \neq 0$. 


\section{REFERENCES}

[Bv1] V. Bavula, Generalized Weyl algebras, kernel and tensor-simple algebras, their simple modules, Canadian Math. Soc. Conf. Proc. 14 (1993), 83-107. CMP 94:09

[Bv2] Global dimension of generalized Weyl algebras, Canadian Math. Soc. Conf. Proc. 18 (1996), 81-107. MR 97e:16018

[B] G. Benkart, Down-up algebras and Witten's deformations of the universal enveloping algebra of $s l_{2}$, Contemporary Math. AMS (to appear).

[BR] G. Benkart and T. Roby, Down-up algebras, J. Algebra (to appear).

[Bj] J.-E. Björk, Filtered Noetherian rings, Noetherian Rings and their Applications, Math. Surveys and Monographs, vol. 24, Amer. Math. Soc., Providence, 1987, pp. 59-97. MR 89c: 16018

[GZ] A. Giaquinto and J. Zhang, Quantum Weyl algebras, J. Algebra 176 (1995), 861-881. MR 96m:16053

[GW] K. R. Goodearl and R. B. Warfield, An Introduction to Noncommutative Noetherian Rings, LMS Student Text 16, Cambridge Univ. Press, Cambridge, 1989. MR 91c:16001

[K] Rajesh S. Kulkarni, Personal communication, 1998.

[L] T. Levasseur, Some properties of non-commutative regular graded rings, Glasgow Math. J. 34 (1992), 277-300. MR 93k:16045

[McR] J. C. McConnell and J. C. Robson, Noncommutative Noetherian Rings, Wiley-Interscience, Chichester, 1987. MR 89j:16023

[Z] Kaiming Zhao, Centers of down-up algebras (to appear).

Department of Mathematics, Wake Forest University, Winston-Salem, North CarOLINA 27109

E-mail address: kirkman@mthcsc.wfu.edu

Department of Mathematics, University of Wisconsin-Milwaukee, Milwaukee, WisCONSIN 53201

E-mail address: musson@csd.uwm.edu

Department of Mathematics, University of Wisconsin-Madison, Madison, Wisconsin 53706

E-mail address: passman@math.wisc.edu 